\title{
A Frequency Domain Approach for Controlling Chaos in Switching Converters
}

\author{
E. Rodríguez and E.Alarcón \\ Department of Electronic Engineering \\ UPC BarcelonaTech \\ 08034 Barcelona, Spain. \\ Email: enricrv, ealarcon@eel.upc.edu
}

\author{
H.H.C Iu \\ School of EECE \\ The University of Western Australia \\ 6009 WA, Australia \\ Email: herbert@ee.uwa.edu.au
}

\author{
A. El Aroudi \\ Department of Electronics and Automatics \\ Universitat Rovira i Virgili \\ 43007 Tarragona, Spain \\ Email: abdelali.elaroudi@urv.net
}

\begin{abstract}
The purpose of this paper is the synthesis from the frequency domain standpoint of a controller for switching power converters with the aim to eliminate bifurcation and chaotic behavior. Firstly the paper analyzes the frequency response of previous delay-based chaos controllers unveiling that they are based in comb-filtering at multiples of the sub-harmonic half of the switching frequency. Secondly, chaos control is explored by using both a single notch filter and a bandstop filter at half of the switching frequency. It is demonstrated that the latter achieves chaos rejection while being an implementation-aware simplification of delay-based methods.
\end{abstract}

\section{INTRODUCTION}

Switching power converters are used in a wide range of power management applications due to their potential for high efficiency and size reduction, despite they tendency to exhibit instabilities. Keeping high efficiency whilst guaranteeing stability is becoming challenging, especially for the new trends in power management which demands further miniaturization and even on-chip integration of switching power converters. This requires to reduce the reactive components size, leading to an increase of switching frequency in order to keep the same dynamics, which is in turn limited due to efficiency constraints. This limitation results in an increase of converter ripples rendering the converter to be more prone to exhibit the so-called fast-scale chaotic instabilities.

In past less demanding applications, the dynamics modeling of such converters has hitherto been carried out by means of average models [1], which are based on a design-oriented circuit-perspective facilitating the synthesis of conventional controllers in the frequency domain. However, these models are inherently limited to only predict the so-called slow-scale instabilities since they do not fully take into account fast dynamics.

Conversely, such fast-scale instabilities have been predicted from a mathematical standpoint by deriving discrete-time models [2]-[4], which despite having excellent prediction accuracy, lack a design-oriented circuit-based perspective.

Fast-scale instabilities or chaos control approaches have been widely explored previously from different standpoints [5]. One of the most relevant chaos control methods is the socalled time-delay feedback control (TDFC or TDAS) [6], [7] or its further extensions (ETDAS) [8]. Apart from the difficulty of implementing continuous-time delays, this set of chaos

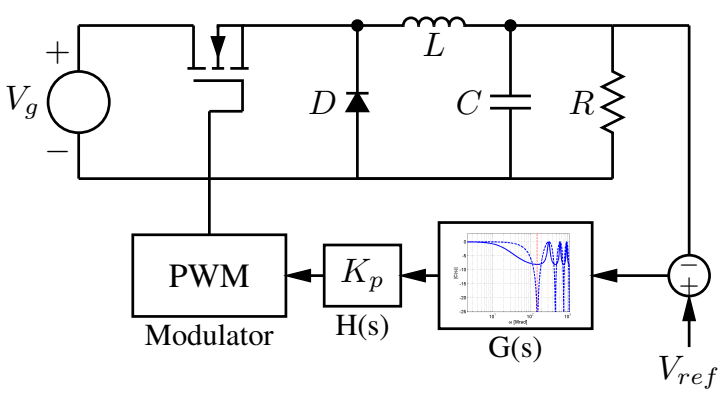

Figure 1. Buck converter under voltage-mode proportional controller $\left(k_{p}\right)$ and constant frequency PWM along with a chaos controller G(s)

controllers are either analyzed by studying their impact upon the discrete-time maps, or by directly characterizing the effect of each chaos control parameter upon the converter stability, difficulting the controller design itself.

This work deals with the synthesis of alternative chaos controllers, derived from a frequency domain interpretation of delay-based controllers, with the ultimate aim of simplifying their design and implementation.

Complementarily to the aforementioned discrete-time modeling for predicting fast-scale instabilities, some recent investigations lead to quantitatively relate the fast-scale stability occurrence boundary to the ripple at the input of the modulator $\left(\rho_{F S}\right)$ [9], providing a design-oriented criterion for predicting fast-scale instabilities which compresses the whole parameter space into a single instability index. Therefore, such ripple index has been taken as a sweep parameter in the bifurcation diagrams used in this work. The stability boundary improvement in terms of fast-scale instability $\Delta \rho$ will be used as a merit figure:

$$
\Delta \rho=\frac{\rho_{c}}{\rho_{c 0}}
$$

being $\rho_{c}$ and $\rho_{c 0}$ the ripple stability boundary with and without the chaos controller $G(s)$, respectively.

This work considers a voltage-mode controlled DC-DC buck converter with constant frequency PWM and proportional control as it is shown in Fig.1 with parameter values: $V_{g}=3$ $\mathrm{V}, V_{\text {ref }}=1.5 \mathrm{~V} L=40 \mathrm{nH}, C=50 \mathrm{nF}, R=1 \Omega, f_{s}=25$ $\mathrm{MHz}$, which are representative of a low-power miniaturized 


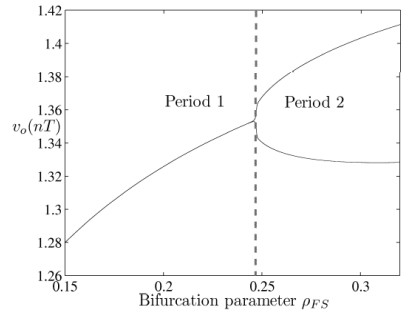

Figure 2. Bifurcation diagram showing period doubling route to chaos by increasing $\rho_{F S}$ in a Buck converter. $\rho_{c 0}=0.2475$

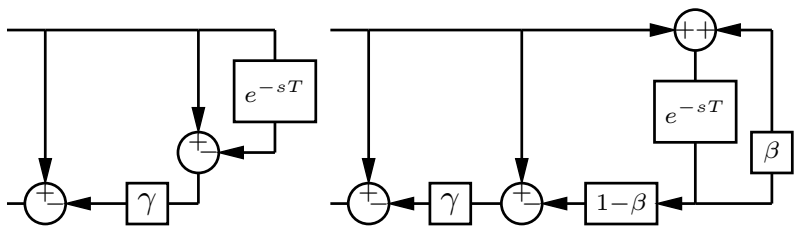

Figure 3. Simple and extended Time-delay feedback control scheme

converter, being $\rho_{c 0} \approx 0.25$.

The evolution of such converter dynamic behavior in terms of the stability index $\rho_{F S}$ is shown in Fig. 2. A perioddoubling bifurcation scenario is clearly observed giving rise to sub-harmonic oscillations and ending up in chaotic behavior. This figure was obtained by sweeping the controller gain, and in turn ripple $\rho_{F S}$, and plotting a steady state representative state variable (capacitor voltage) sampled at the switching frequency rate.

The remaining of the paper is structured as follows. First, Section II explores the frequency response of delay-based controllers as well as the effect of their parameters on stability boundaries. Subsequently, Section III demonstrates that by modifying the controller frequency band around half of the switching frequency through bandstop filters, control of fast scale instability while simplifying the implementation is feasible.

\section{TIME-DELAY FEEDBACK CHAOS CONTROLLERS (TDFC)}

This section revisits time-delay feedback chaos controllers, which are based on adding a time-delay module, as it is shown in Fig. 3. The extended TDFC scheme includes an additional feedback inner loop, added to the delayed variable. Their Laplace domain transfer function can be expressed as:

$$
G(s)=1-\gamma \frac{e^{s T}-1}{e^{s T}-\beta}
$$

where $\beta$ is zero for the TDFC and it takes a non-null value in the case of extended TDFC.

From the frequency responses of both controllers (Fig. 4), it is possible to observe that they implement a comb-filtering function located at half of the switching frequency and its harmonics, whereas it has no effect at the switching frequency, hence demonstrating the non-invasive nature of such delaybased controllers. Such behavior in frequency domain unveils
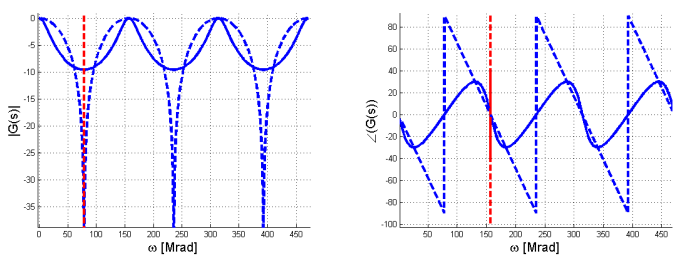

Figure 4. Frequency response of $G(s)$ with $\beta=0$ (TDFC, dashed-line) and $\beta=0.5$ (extended TDFC, solid-line) $\gamma=0.5$ showing attenuation at half of switching frequency (vertical dashed-line)

that chaos control is carried out by avoiding the exhibition of sub-harmonic dynamics.

Regarding the design-space of TDFC chaos controllers, two parameters are identified, namely $\gamma$ and $\beta$. Numerical simulations show that only some ranges of $\gamma$ values are valid to avoid instabilities. By increasing the $\gamma$ value, the stability margin in terms of fast-scale is improved, being $\gamma_{F S}$ the minimum value required to avoid such instabilities. On the other hand, the higher $\gamma$ is, the closer the system is to the slow scale stability boundary, exhibiting Hopf instabilites when $\gamma$ reaches a critical value $\gamma_{S S}$ as it is shown in Fig. 5.
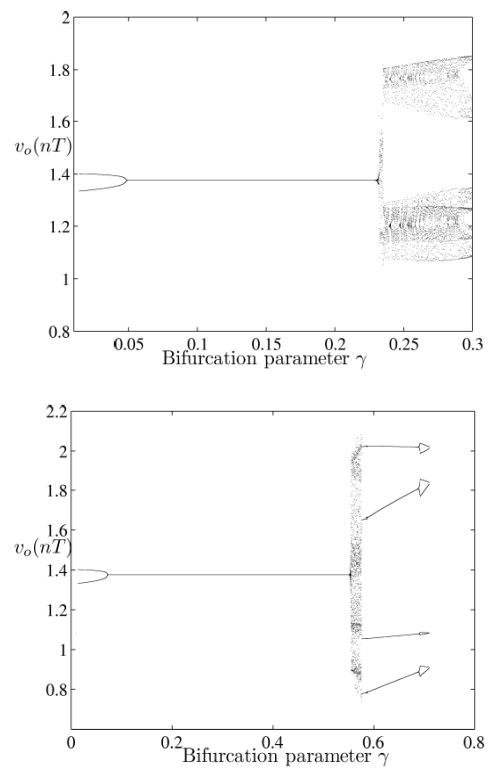

Figure 5. Bifurcation diagram resulting in Hopf instability by increasing parameter $\gamma$ of TDFC and ETDFC (with $\beta=0.5$ ). $\gamma_{F S, T D F C}=0.05$ $\gamma_{S S, T D F C}=0.21 \gamma_{F s, E T D F C}=0.08 \gamma_{S S, E T D F C}=0.55$ (simulated for $\rho_{F S}=0.2813$ )

The dynamic behavior in Fig. 5 shows that the major effect of the $\beta$ parameter in the extended TDFC case is to shift away the border of occurrence of Hopf bifurcation $\gamma_{S S}$ due the smoothing of the phase response. Fig. 6 shows that the extended TDFC case, which is slow scale stable for higher $\gamma$ values, can benefit of such $\gamma$ higher values to achieve better fast scale stability margins.

Regardless of the advantage of the extended TDFC, the main drawback of using a delay still holds, thereby compromising 

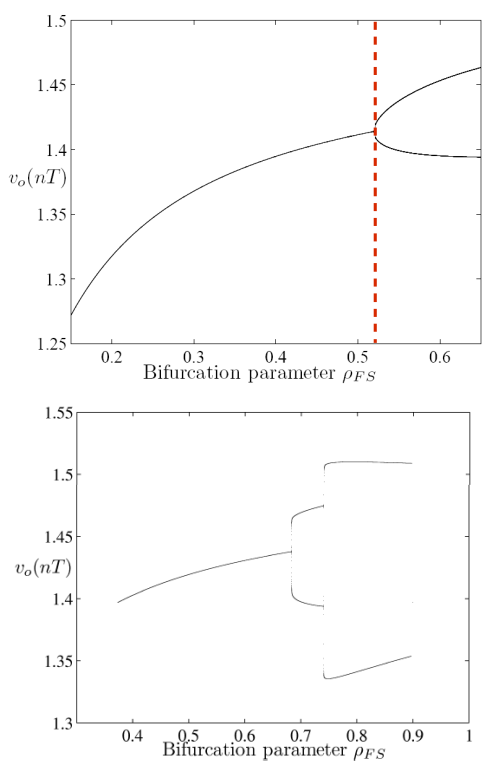

Figure 6. Bifurcation diagram in terms of $\rho_{F S}\left(k_{p}\right)$ with TDFC $(\beta=0$ $\gamma=0.15) \Delta \rho=202 \%$ and with extended TFDC ( $\beta=0.5 \gamma=0.3) \Delta \rho=278 \%$

its implementability.

\section{NOTCH AND BAND-STOP FILTER CHAOS CONTROLLER}

The controllers analyzed in the previous section are based on eliminating the sub-harmonic components from the feedback path in order to reduce their effect on fast-scale dynamics. However, it will be shown in this section that it is enough to attenuate the first sub-harmonic (at half of the switching frequency) to obtain a successful control of chaos.

A direct way to attenuate sub-harmonic frequency is by means of a bandstop filter, the general transfer function of which is given by:

$$
G(s)=\frac{s^{2}+2 \xi_{1} \omega_{n} s+\omega_{n}^{2}}{s^{2}+2 \xi_{2} \omega_{n} s+\omega_{n}^{2}}
$$

where $\omega_{n}=\pi f_{s}$ (half of the switching frequency) and $\mathbf{Q}=\left(2 \xi_{2}\right)^{-1}$. Note that for $\xi_{1}=0$, the transfer function is a pure notch filter centered at half of the switching frequency.

The bifurcation diagram in Fig. 7 shows that by using such ideal notch filter controller $\left(\xi_{1}=0\right)$, the stability boundary can not improve due to the previous exhibition of a quasiperiodic slow-scale instability. Despite the fast-scale boundary is shifted away, a slow-scale instability appears, thereby reducing the overall stability margin.

This behavior can also be shown by deriving the discretetime map of the controlled converter (see Appendix), the evolution of which is shown in Fig. 8. The diagram shows that the notch filter produces the eigenvalues to cross the unit circle with imaginary values (which is indicative of slow-scale instability) during an interval after which they end up in real negative values (which is indicative of fast-scale instability)

Analogously to the extended TDFC case, a band-stop filter $\left(\xi_{1}>0\right)$ is used instead of a pure notch, hence leading to
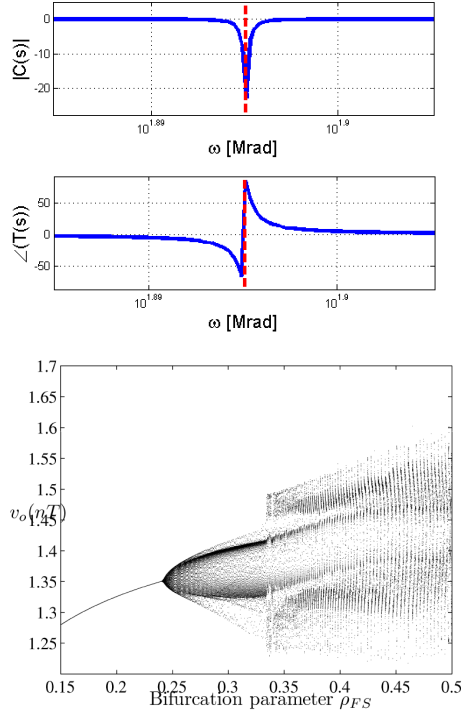

Figure 7. Notch controller with $\mathrm{Q}=500\left(\xi_{1}=0\right.$ and $\left.\xi_{2}=0.001\right)$ frequency representation (Bode plot), centered at half of switching frequency (vertical dashed-line) and bifurcation diagram by sweeping $\rho_{F S}\left(k_{p}\right)$. FS instability improvement $\Delta \rho=96 \%$

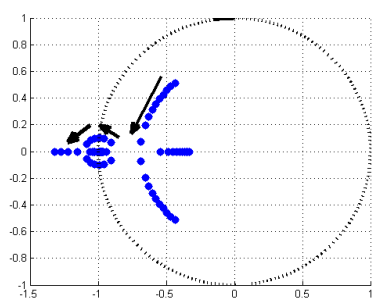

Figure 8. Eigenvalues of the Jacobian derived from discrete-time map of the buck converter with a notch filter $\left(\xi_{2}=0.001\right)$ by sweeping $\rho_{F S}$ as in Fig. 7

smoothing the frequency response in terms of both magnitude and phase, therefore maintaining the system farther away from the slow-scale instability boundary, as quantitatively indicated in an increase of $\Delta \rho=204 \%$.

The Bode response of the band-stop filter and the route to chaos diagram are shown in Fig. 9. Note that the trajectory of the eigenvalue shown in Fig. 10 is different from the pure notch trajectory, since they cross the unit circle in -1 , hence leading to period-2 bifurcation alone.

By properly adjusting the values of $\xi_{1}$ and $\xi_{2}$, a similar stability boundary improvement as TDFC is reached in terms of $\Delta \rho$, but simplifying the implementation regarding delaybased controllers.

\section{CONClusions}

This paper explores chaos control methods for switching power converters from a frequency domain standpoint, with the goal of synthesizing alternative controllers with simpler implementation. The work has firstly revisited, in the frequency domain, previously proposed chaos controllers (TDFC 

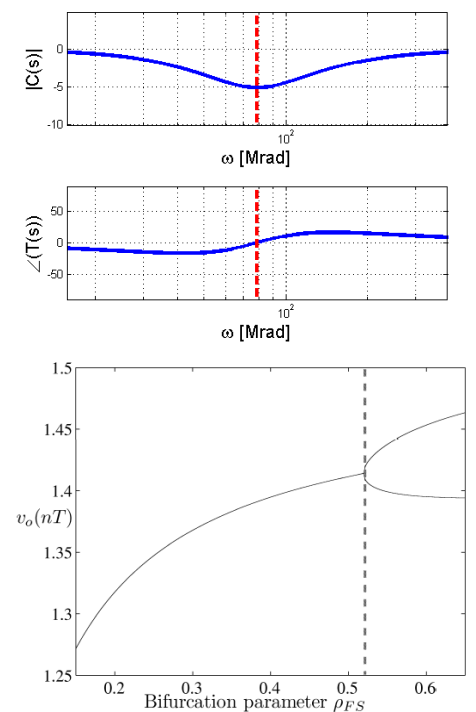

Figure 9. Bandstop controller frequency representation (Bode plot) centered at half of switching frequency (vertical dashed-line), and bifurcation diagram by sweeping $\rho_{F S}\left(k_{p}\right)$ and $\xi_{1}=0.5, \xi_{2}=0.9$. FS instability improvement $\Delta \rho=204 \%$

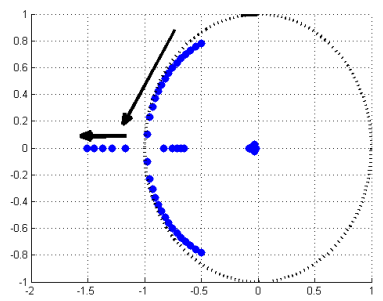

Figure 10. Eigenvalues of Jacobian derived from discrete-time map of the buck converter with a bandstop filter $\left(\xi_{1}=0.5, \xi_{2}=0.9\right)$ by sweeping $\rho_{F S}$ as in Fig.9

and its extended version), thereby showing that they are based on comb-filtering at multiples of sub-harmonic frequencies. Subsequently, a notch filter at half of the switching frequency is proposed as an implementation-aware simplification of time-delay-based controllers. Such narrowband tuned bandpass filter compensator effectively controls fast-scale perioddoubling, but incurs in making the switching regulator more prone to exhibit slow-scale instability. Consequently, a bandstop filter is finally proposed as an implementation-aware effective chaos controller. The study of both alternatives has been carried out by bifurcation diagrams from the instantaneous converter equations contrasted to the predictions from the corresponding discrete-time maps.

\section{ACKNOWLEDGEMENT}

This work is partially supported by an FPU-grant, project TEC-2007-67988-C02-01,02, and project RUE CSD200900046, Consolider-Ingenio 2010 Programme of Ministry of Science and Innovation of Spain.

\section{ANNEX: NOTCH AND BAND-STOP FILTER DISCRETE-TIME MAPS}

In order to derive the discrete-time map for a buck converter with either a notch or a band-stop filter, the additional secondorder dynamics of the chaos control filters are added to the converter dynamics, thereby increasing to fourth order the order of the complete closed-loop system dynamics expressed by the state equations in each switching interval $(\dot{x}=A x+B)$.

The discrete-time map for such system can be expressed as:

$$
\begin{aligned}
& P\left(x_{n}\right)=X_{e q, 2}+\cdots \\
& \quad+\phi_{2}\left(T-d_{n} T\right)\left(X_{e q, 1}+\phi_{1}\left(d_{n} T\right)\left(x_{n}-X_{e q, 1}\right)-X_{e q, 2}\right)(4)
\end{aligned}
$$

where $X_{e q, i}=-A_{i}^{-1} B_{i}$ is the equilibrium point of the linear configuration $i(i \in\{1,2\})$ and $\phi_{i}\left(t_{i}\right)=e^{A_{i} t_{i}}$.

Additionally, the switching surface, which depends upon the controller law and the modulator (PWM with saw-tooth $h(t)$ ) can be expressed as:

$$
\sigma(t)=\mathbf{K}\left[X_{e q, 1}+\phi_{1}\left(d_{n} T\right)\left(x_{n}-X_{e q, 1}\right)\right]-h(t)
$$

where $\mathbf{K}=\left(\begin{array}{llll}-k_{p} & 0 & 0 & k_{p}\left(\xi_{2}-\xi_{1}\right) 2 w_{n} L_{n}\end{array}\right)$, being $w_{n}$ the center frequency of the notch filter.

The stability analysis is carried out by studying the local behavior of the map in the vicinity of steady-state $\left(x^{*}\right)$, thereby extracting a Jacobian matrix $D P$, the eigenvalues of which describe the amount of expansion and contraction:

$$
D P=\frac{\delta P}{\delta x_{n}}-\frac{\delta P}{\delta d_{n}}\left(\frac{\delta \sigma}{\delta d_{n}}\right)^{-1} \frac{\delta \sigma}{\delta x_{n}}
$$

\section{REFERENCES}

[1] R. Middlebrook and S. Cuk, "A general unified approach to modelling switching-converter power stages," in Power Electronics Specialists Conference, Cleveland, Ohio, June 8-10, 1976, Record, June 1976, pp. 18-34.

[2] D. C. Hamill and D. J. Jeffries, "Subharmonics and chaos in a controlled switched-mode power converter," IEEE Transactions on Circuits and Systems, vol. 35, no. 8, pp. 1059-1061, Aug. 1988.

[3] C. Tse, "Chaos from a buck switching regulator operating in discontinuous mode," Int. J. Circuit Theory Applicat., vol. 22, no. 6, pp. 263-278, Aug. 1994.

[4] M. di Bernardo, F. Garefalo, L. Glielmo, and F. Vasca, "Switchings, bifurcations, and chaos in DC/DC converters," Circuits and Systems I: Fundamental Theory and Applications, IEEE Transactions on, vol. 45, no. 2, pp. 133-141, feb 1998.

[5] G. Chen, G. Chen, and X. Dong, "Control of chaos-a survey," in Decision and Control, 1993., Proceedings of the 32nd IEEE Conference on, X. Dong, Ed., 1993, pp. 469-474 vol.1.

[6] K. Pyragas, "Continuous control of chaos by self-controlling feedback," Physics Letters A, vol. 170, pp. 421-428, Nov. 1992.

[7] C. Batlle, E. Fossas, and G. Olivar, "Time-delay stabilization of the buck converter," in Control of Oscillations and Chaos, 1997. Proceedings., 1997 1st International Conference, vol. 3, 1997, pp. 590-593 vol.3.

[8] K. Pyragas, "Control of chaos via extended delay feedback," Physics Letters A, vol. 206, no. 5-6, pp. 323 - 330, 1995.

[9] E. Rodriguez, G. Villar, F. Guinjoan, A. Poveda, A. El Aroudi, and E. Alarcon, "General-purpose ripple-based fast-scale instability prediction in switching power regulators," pp. 2423-2426, May 2007. 